\title{
Premarket evaluation of Monofluor reagent for detecting Chlamydia trachomatis in adolescent outpatients
}

\author{
L E PHILlipS, S FARO, P B SMITH, M G MARTENS, G D RIDDLE, \\ K H GOODRICH
}

From the Department of Obstetrics and Gynecology, Baylor College of Medicine, Houston, Texas, USA

SUMMARY A new direct fluorescent antibody reagent, Monofluor, was evaluated for detecting Chlamydia trachomatis in fresh specimens. Monofluor was compared with MicroTrak and with cultivation in McCoy cells. Both direct systems were slightly less sensitive than culture, but no significant differences in specificity or sensitivity were noted between culture, Monofluor, or MicroTrak results.

Chlamydia trachomatis is one of the most prevalent sexually transmitted organisms and is responsible for at least $20 \%$ of cases of pelvic inflammatory disease in some patient populations. ' Endometritis and salpingitis due to $C$ trachomatis are often referred to as the silent infections. In many instances, the patient does not know that she has an infection until she is evaluated for infertility. The need for efficient and reliable detection methods is therefore imperative if treatment is to be instituted in patients who are at risk for asymptomatic infection with $C$ trachomatis. Currently available techniques for detecting $C$ trachomatis include cultivation of the organism in tissue cell cultures, antigen detection by enzyme linked immunoassay, and fluorescent antibody staining. The most commonly used fluorescent antibody technique has been MicroTrak (Syva Company, Palo Alto, California, USA).

We conducted a comparative evaluation of Monofluor (Synbiotics, San Diego, California, USA), MicroTrak, and tissue culture. The high incidence of positivity for $C$ trachomatis in the asymptomatic population studied emphasises the need for rapid and reliable diagnostic alternatives.

\section{Patients, materials, and methods}

STUDY POPULATION

We screened 116 sexually active adolescent girls aged 13 to 19 who attended a Teen Health Clinic at

Address for reprints: Dr S Faro, Department of Obstetrics and Gynecology, Baylor College of Medicine, Houston, Texas, USA

Accepted for publication 30 September 1987
Jefferson Davis Hospital in Houston, Texas, from 13 May to 6 June 1986. Eight patients were white, 21 hispanic, and 87 black. Most were indigent and lived in the inner city. Ninety three were attending the clinic for family planning counselling and yearly pelvic examination or for postpartum examinations.

\section{COLLECTING AND PROCESSING SPECIMENS}

Two endocervical specimens were collected from each patient on sterile calcium alginate swabs after the tip of the cervix had been cleansed of vaginal discharge and debris with a large cotton tipped applicator. The first swab was used to make smears of the sample within the perimeter of two circular areas (wells) located at opposite ends of a glass slide, one end of which was frosted. The slide was allowed to air dry and then fixed in absolute methanol for 10 minutes. It was removed from the methanol, allowed to air dry, fixed, and frozen at $-70^{\circ} \mathrm{C}$. Slides were stained in batches of 20 within one week after preparation.

A second swab was placed in chlamydial transport medium and kept refrigerated (at $2-8^{\circ} \mathrm{C}$ ) until delivered to the laboratory within 18 hours. On receipt in the laboratory, each transport tube was vortexed for 30 seconds to remove cellular material from the swab. Excess moisture was removed from the swab by pressing it against the side of the tube as it was withdrawn. The swab was then discarded. The tubes were centrifuged at $800 \times g$ for five minutes to remove debris. The overlay medium was removed from commercially prepared McCoy cell monolayers grown on cover slips contained in 1 fluid dram $(3.7 \mathrm{ml})$ vials. A $0.5 \mathrm{ml}$ volume of the inoculum was added, the vials were centrifuged for one hour at $3000 \times g$ at $30^{\circ} \mathrm{C}$, and 
then incubated at $35^{\circ} \mathrm{C}$ for one hour. Fresh isolation medium was added, and the vials were incubated for 48-72 hours before staining. The chlamydial transport medium, McCoy cell monolayers, and isolation medium were obtained from Bartels Immunodiagnostics (Bellevue, Washington, USA).

\section{STAINING AND INTER PRETATION}

The well nearest the frosted end of each slide was stained with the Monofluor reagent, and the other well was stained with the MicroTrak reagent. A $\mathbf{4 0} \mu \mathrm{l}$ drop of the Monofluor reagent or a $30 \mu \mathrm{l}$ drop of the Syva reagent was added to the appropriate well and spread over the entire surface of the specimen. The slides were incubated for 15 minutes in a humidified chamber at room temperature. They were then removed from the chamber and the edges were gently tapped on absorbent paper to remove excess reagent. The slides were rinsed by gentle swirling in deionised water for 10 seconds. The edges of the slides were gently tapped on absorbent paper and excess water was carefully removed from the area around each specimen. One drop of mounting fluid was added to the centre of each well, and a coverslip was added.

All the specimens were examined with a Nikon Labophot EPI fluorescent microscope with an IF 535550 interference excitation filter, DM575 dichroic mirror, and $580 \mathrm{~W}$ barrier filter. The well containing the specimen stained with Monofluor was read by one technologist, and the well containing the specimen stained with MicroTrak was read by another. Neither technologist knew the results obtained by the other reagent or the culture results, though both rechecked discrepancies. Specimens were scanned at $400 \times$ magnification for the presence of counterstained epithelial cells. Only slides containing adequate cellular material (at least 20 parabasal, basal, or columnar epithelial cells) were included in the analysis. The slides were then scanned for apple green elementary bodies (or more rarely reticulate bodies). Elementary bodies were round with smooth edges and were about $0.2 \mu \mathrm{m}$ in diameter. The presence of elementary bodies was confirmed at $1000 \times$ magnification. Each well was given a score $(0,<10,<100$, or $>100)$ according to the number of elementary bodies observed. Specimens were considered to be positive if there were at least two elementary bodies present. All positive specimens, however, contained at least five elementary bodies. Background fluorescence was given a score on a scale of $\mathbf{0}$ (no background fluorescence) to ++ (moderate background fluorescence).

The McCoy cell monolayers were stained with the Ortho culture confirmation reagent (Ortho Diagnostics, Raritan, New Jersey, USA) and by the Synbiotics culture confirmation reagent. Both culture staining systems required the removal of growth media, fixa- tion with methanol for 10 minutes, removal of methanol, adding one drop of fluorescein labelled antibody to $C$ trachomatis to the monolayer, incubating for 30 minutes at $37^{\circ} \mathrm{C}$ in a humidified chamber, rinsing, and mounting the coverslip cell-side-down on a clean glass slide. The stained monolayers were scanned at $250 \times$ to $400 \times$ magnification for typical apple green inclusion bodies, which measured 8 to $15 \mu \mathrm{m}$ in diameter. It was also possible to detect the smaller elementary bodies, if the inclusions had ruptured, by the use of $1000 \times$ magnification. Blind subcultures were not performed.

The method used for comparing results was that described by Galen. ${ }^{2}$

\section{Results}

Of the 116 culture specimens, 29 yielded $C$ trachomatis. All 116 slides contained adequate cellular material for comparison, but two stained with the MicroTrak reagent and four stained with Monofluor were uninterpretable due to excessive background fluorescence. Three specimens yielded a toxic and uninterpretable culture result. Thus a total of 107 specimens were compared by the three systems.

Table 1 compares detection by the three systems. Of the 107 specimens examined, two were negative by the Monofluor reagent that were positive by culture and MicroTrak. All specimens positive by Monofluor were also positive by MicroTrak. Six specimens positive by culture were negative by both direct systems.

Five specimens were negative by culture and positive by both MicroTrak and Monofluor. Four of these contained fewer than 10 elementary bodies and the fifth contained 10-100 elementary bodies. Table 2 shows that, if these five specimens were considered to be true positives (false negative by culture), the sensitivity of culture would be $84 \%$, that of Monofluor $75 \%$, and that of MicroTrak $81 \%$. The specificity and predictive value of a positive result by all three systems would then be $100 \%$. The predictive values of a

Table 1 Comparison of Monofluor and MicroTrak with culture results for detecting Chlamydia trachomatis in 107 endocervical specimens

\begin{tabular}{|c|c|c|c|c|}
\hline \multirow[b]{2}{*}{ Culture } & \multicolumn{2}{|c|}{ Monofluor } & \multicolumn{2}{|c|}{ MicroTrak } \\
\hline & Positive & Negative & Positive & $\overline{\text { Negative }}$ \\
\hline $\begin{array}{l}\text { Positive } \\
\text { Negative }\end{array}$ & $\begin{array}{r}19 \\
5\end{array}$ & $\begin{array}{r}8 \\
75\end{array}$ & $\begin{array}{r}21 \\
5\end{array}$ & $\begin{array}{r}6 \\
75\end{array}$ \\
\hline $\begin{array}{l}\text { Sensitivity } \\
\text { Specificity } \\
\text { Positive } \\
\text { predictive value } \\
\text { Negative } \\
\text { predictive value }\end{array}$ & $\begin{array}{l}70^{\circ} \\
94^{\circ} \\
79^{\circ} \\
90^{\circ}\end{array}$ & $\begin{array}{l}(19 / 27) \\
(75 / 80) \\
(19 / 24) \\
(75 / 83)\end{array}$ & \multicolumn{2}{|c|}{$\begin{array}{l}78 \%(21 / 26) \\
94 \%(21 / 27)\end{array}$} \\
\hline
\end{tabular}


Table 2 Results obtained by culture, MicroTrak, and Monofluor compared with confirmed positive results*

\begin{tabular}{|c|c|c|c|c|c|c|}
\hline & \multicolumn{2}{|l|}{ Culture } & \multicolumn{2}{|c|}{ Monofluor } & \multicolumn{2}{|c|}{ MicroTrak } \\
\hline & Positive & Negative & Positive & Negative & Positive & Negative \\
\hline $\begin{array}{l}\text { Confirmed results: } \\
\text { Positive } \\
\text { Negative }\end{array}$ & $\begin{array}{r}27 \\
0\end{array}$ & $\begin{array}{r}5 \\
75\end{array}$ & $\begin{array}{r}24 \\
0\end{array}$ & $\begin{array}{r}8 \\
75\end{array}$ & $\begin{array}{r}26 \\
0\end{array}$ & $\begin{array}{r}6 \\
75\end{array}$ \\
\hline $\begin{array}{l}\text { Sensitivity } \dagger \\
\text { Specificity } \\
\text { Positive predictive value } \\
\text { Negative predictive value } \dagger\end{array}$ & \multicolumn{2}{|c|}{$\begin{array}{r}84 \%(27 / 32) \\
100 \%(75 / 75) \\
100 \%(27 / 27) \\
94 \%(75 / 80)\end{array}$} & \multicolumn{2}{|c|}{$\begin{array}{c}75 \%(24 / 32) \\
100 \%(75 / 75) \\
100 \%(24 / 24) \\
90 \%(75 / 83)\end{array}$} & \multicolumn{2}{|c|}{$\begin{array}{c}81 \%(26 / 32) \\
100 \%(75 / 75) \\
100 \%(26 / 26) \\
93 \%(75 / 81)\end{array}$} \\
\hline
\end{tabular}

*Five specimens positive by MicroTrak and Monofluor were counted as true positives (culture misses).

†Differences not significant by $\chi^{2}$ test.

Table 3 Monofluor compared with MicroTrak for detecting Chlamydia trachomatis in 107 endocervical specimens

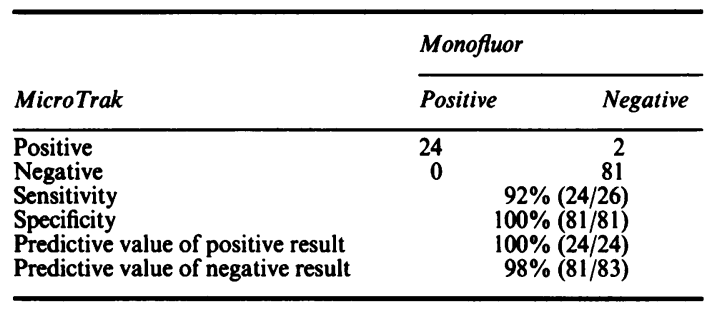

negative result would be $94 \%$ by culture, $90 \%$ by Monofluor, and $93 \%$ by MicroTrak.

Table 3 shows that, when compared with MicroTrak, Monofluor had a sensitivity of $92 \%$, specificity of $100 \%$, positive predictive value of $100 \%$, and negative predictive value of $98 \%$.

Three specimens stained with MicroTrak and 15 stained with Monofluor showed a + level of background fluorescence. One specimen stained with Monofluor showed a ++ level of background fluorescence.

There were no discrepant results between monolayers stained with the Ortho fluorescent antibody reagent and those stained with Monofluor.

\section{Discussion}

The cost, labour, and potential lack of sensitivity due to loss of viability in delayed transport of specimens has made procedures other than cultivation for $C$ trachomatis desirable. Some investigators who compared MicroTrak with culture results have found an acceptable level of performance with that reagent. ${ }^{3-5}$ We undertook the present study to compare the methodology and ease of interpretation of a new reagent, Monofluor, with that of MicroTrak and to compare both fluorescent stains with our standard cell culture technique.

Monofluor, MicroTrak, and the Ortho culture confirmation reagent all contain monoclonal antibodies directed against the major outer membrane protein (MOMP) of $C$ trachomatis. The Ortho reagent also contains monoclonal antibodies against the lipopolysaccharide (LPS) portion of the cell wall. The presence of two antibodies in the Ortho reagent offered no apparent increase in sensitivity, as all the specimens positive by the Ortho reagent were also positive by the Monofluor reagent. The discrepancies between the direct tests and culture results may have been due to sampling error or inappropriate storage of the transport tube after specimen collection. The latter would result in loss of viability and hence a negative culture, whereas the direct tests would have given positive results due to staining of antigen. Blind subculture of initially negative specimens was not performed, as we have not found that to increase positive cultures. Thus we have found that the large inoculum size, the high speed of centrifugation, and use of a fluorescent antibody reagent (rather than iodine) for staining provides optimum detection of $C$ trachomatis.

The results obtained with both fluorescent reagents were comparable to those obtained by other investigators who compared results of MicroTrak with culture results from asymptomatic female patients. ${ }^{4-9}$ The culture procedures used by different investigators have varied somewhat, making it difficult to make clear cut comparisons with other studies. Some investigators used McCoy cell monolayers contained in 96 well microtitre plates. ${ }^{6-8}$ Stary et al used the MicroTrak culture confirmation stain for staining McCoy cells, ${ }^{6}$ whereas Quinn et al and Chernesky et al used iodine for staining McCoy cells. ${ }^{78}$ Other investigators used McCoy cell monolayers contained in one dram vials for culture.$^{49}$ Uyeda et al used the MicroTrak culture confirmation stain, ${ }^{4}$ whereas Lipkin et al used iodine for staining McCoy cells. ${ }^{9}$ Fluorescent staining of McCoy cells has been shown to be more sensitive than iodine staining in detecting positive cultures. ${ }^{70} 11$ Furthermore, cultivation in vials has been shown to be more sensitive than in microtitre plates for recovering C trachomatis. $^{912}$ 
Perhaps the most accurate results of comparisons are obtained by comparing new reagents or test systems with the reference method under optimum conditions, which is cultivation in vials and staining with fluorescent antibody reagents. In that respect, the present study most closely resembles that of Uyeda $e t$ $a l^{4}$ with the difference that the Ortho and Monofluor, rather than MicroTrak, fluorescent stains were used to confirm culture. The differences in sensitivity between the study of Uyeda et al $(96 \%)$ and the present study $(78 \%)$ may possibly be explained by differences in specimen collection techniques for culture or direct examination, differences in time between collection of specimens and inoculation of McCoy cells, differences in performance characteristics of the Ortho culture confirmation test and the MicroTrak culture confirmation test, or a combination of these and other factors.

We found that the Monofluor reagent was only slightly less sensitive than the MicroTrak reagent compared with culture results. The other variables considered (specificity and predictive value of positive or negative results) were nearly equal. Both manufacturers recommend that 10 elementary bodies should be seen after staining the specimen directly for a result to be considered positive. We found, however, that the size, shape, and colour of elementary bodies stained with either reagent were easily recognised even when present in small numbers. To use 10 as the cutoff point would unnecessarily decrease the sensitivity of both tests. Although the technical procedure of Monofluor was as simple as MicroTrak, Monofluor stained specimens were slightly more difficult to interpret because of background fluorescence. This difficulty was overcome with practice, but may prove quite frustrating and fatiguing to technologists not accustomed to reading fluorescence.

The prevalence of infection with $C$ trachomatis imposes the necessity of diagnostic techniques for recognition of that organism even in settings where cultivation is not available. Both fluorescent reagents studied are designed to be interpreted within 40 minutes after collection of the specimen. Slides are usually sent to a laboratory and stained in batches, however, so the results are often not available until after the patient has left. Furthermore, direct antigen detection is reliable in patient populations where the incidence of $C$ trachomatis is high. ${ }^{79}$ Such settings include sexually transmitted disease (STD) clinics or other large scale screening facilities that may process over 20 specimens a day. As pointed out by other investigators, ${ }^{13}$ accurate interpretation of fluorescent stained slides requires well trained microscopists. The efficiency of even the best trained microscopists will diminish with large numbers of slides to read. ${ }^{14}$ Consequently, we consider the best setting for direct fluorescent staining to be a high prevalence population of low volume. In those settings Monofluor offers an alternative to the MicroTrak reagent.

\section{References}

1 Eschenbach DA. New concepts of obstetrics and gynecologic infection. Arch Intern Med 1982;142:2039-40.

2 Galen RS. Predictive value theory. Diagnostic Medicine. 1979;1:23-31.

3 Thomas BJ, Evans RT, Hawkins DA, Taylor-Robinson D. Sensitivity of detecting Chlamydia trachomatis elementary bodies in smears by use of a fluorescein labelled monoclonal antibody: comparison with conventional chlamydial isolation. $J$ Clin Pathol 1984;37:812-6.

4 Uyeda CT, Welborn P, Ellison-Bireng N, Shunk K, Tsaouse B. Rapid diagnosis of chlamydial infections with the MicroTrak direct test. J Clin Microbiol 1984;20:948-50.

5 Williams T, Maniar AC, Brunham RC, Hammond GW. Identification of Chlamydia trachomatis by direct immunofluorescence applied in specimens originating in remote areas. $J$ Clin Microbiol 1985;22:1053-4.

6 Stary A, Kopp W, Gebhart W, Söltz-Szöts J. Culture versus direct specimen test: comparative study of infection with Chlamydia trachomatis in Viennese prostitutes. Genitourin Med 1985;61:258-60.

7 Quinn TC, Warfield P, Kappus E, Barbacci M, Spence M. Screening for Chlamydia trachomatis infection in an inner city population: a comparison of diagnostic methods. J Infect Dis 1985;152:419-23.

8 Chernesky MA, Mahony JB, Castriciano S, et al. Detection of Chlamydia trachomatis antigens by enzyme immunoassay and immunofluorescence in genital specimens from symptomatic and asymptomatic men and women. J Infect Dis 1986;154:1418.

9 Lipkin ES, Moncada JV, Shafer M-A, Wilson TE, Schachter J. Comparison of monoclonal antibody staining and culture in diagnosing cervical chlamydial infection. J Clin Microbiol 1986;23:114-7.

10 Stamm WE, Tam M, Koester M, Cles L. Detection of Chlamydia trachomatis inclusions in McCoy cell cultures with fluoresceinconjugated monoclonal antibodies. J Clin Microbiol 1983; 17:666-8.

11 Zapata M, Chernesky M, Mahony J. Indirect immunofluorescence staining of Chlamydia trachomatis inclusions in microculture plates with monoclonal antibodies. J Clin Microbiol 1984;19:937-9.

12 Smith TF. Comparative recoveries of Chlamydia from urethral specimens using glass vials and plastic microtiter plates. $\mathrm{Am} \mathrm{J}$ Clin Pathol 1976;67:496-8.

13 Mallinson H, Turner GC, Carey PB, Khan MH. Rapid detection of Chlamydia trachomatis with monoclonal antibodies. Lancet 1984;i:1180-1.

14 Foulkes SJ, Deighton R, Feeney ARB, Mohanty KC, Freeman CWJ. Comparison of direct immunofluorescence and cell culture for detecting Chlamydia trachomatis. Genitourin Med 1985;61:255-7. 